\title{
GLOBULAR CLUSTER AND GALAXY FORMATION: M31, THE MILKY WAY, AND IMPLICATIONS FOR GLOBULAR CLUSTER SYSTEMS OF SPIRAL GALAXIES ${ }^{1}$
}

\author{
David Burstein, ${ }^{2}$ Yong Li, ${ }^{2}$ Kenneth C. Freeman, ${ }^{3}$ John E. Norris, ${ }^{3}$ Michael S. Bessell, ${ }^{3}$ \\ Joss Bland-Hawthorn, ${ }^{4}$ Brad K. Gibson, ${ }^{5}$ Michael A. Beasley, ${ }^{5}$ Hyun-chul Lee, ${ }^{5}$ \\ Beatriz Barbuy, ${ }^{6}$ John P. Huchra, ${ }^{7}$ Jean P. Brodie, ${ }^{8}$ and Duncan A. Forbes ${ }^{5}$ \\ Received 2004 May 25; accepted 2004 June 15
}

\begin{abstract}
We find that the globular cluster (GC) systems of the Milky Way and of our neighboring spiral galaxy, M31, comprise two distinct entities, differing in three respects. First, M31 has a set of young GCs, ranging in age from a few times $10^{2}$ Myr to 5 Gyr old, as well as old GCs. No such very young GCs are known in the Milky Way. Second, we confirm that the oldest M31 GCs have much higher nitrogen abundances than do Galactic GCs at equivalent metallicities, while carbon abundances appear normal for the GCs in both galaxies. Third, Morrison and coworkers have shown that M31 has a subcomponent of GCs that follow closely the disk rotation curve of that galaxy. Such a GC system in our own Galaxy has yet to be found. The only plausible scenario for the existence of the young M31 GC comes from the hierarchical-clustering-merging (HCM) paradigm for galaxy formation. We infer that M31 has absorbed more of its contingent of dwarf systems in the recent past than has the Milky Way. This inference has three implications: First, all spiral galaxies could differ in their GC properties, depending on how many companions each galaxy has and when the parent galaxy absorbs them. In this spectrum of possibilities, apparently the Milky Way ties down one end, in which almost all of its GCs were absorbed 1012 Gyr ago. Second, it suggests that young GCs are preferentially formed in the dwarf companions of parent galaxies and then absorbed by the parent galaxy during mergers. Third, the young GCs seen in tidally interacting galaxies might come from the dwarf companions of these galaxies, rather than be made anew in the tidal interaction. However, there is no ready explanation for the marked difference in nitrogen abundance for the stars in the old M31 GCs relative to those in the oldest Galactic GCs, especially the most metal-poor GCs in both galaxies. The predictions made by $\mathrm{Li} \&$ Burstein regarding the origin of nitrogen abundance in GCs are consistent with what is found for the old M31 GCs compared to that for the two 5 Gyr old M31 GCs.
\end{abstract}

Subject headings: galaxies: evolution — galaxies: formation — galaxies: individual (M31) galaxies: star clusters - globular clusters: general

\section{INTRODUCTION}

The most studied globular system in our field of study are the globular clusters (GCs) in our own Galaxy. These clusters have been studied in numerous ways, including detailed modeling of their color-magnitude diagrams (e.g., Bergbusch \& VandenBerg 2001 and references therein) and spectra obtained of their giant stars, horizontal branch stars, and mainsequence stars (e.g., Kraft \& Ivans 2003; Castilho et al. 2000; Carretta et al. 2004; Behr 2003). However, it was not until Li \& Burstein (2003) that it was discovered that in integrated light, the most metal-poor Galactic GCs show relatively strong molecular absorption systems for NH at $3360 \AA$.

\footnotetext{
${ }^{1}$ Observations reported here were obtained at the MMT Observatory, a joint facility of the Smithsonian Institution and the University of Arizona.

2 Department of Physics and Astronomy, Arizona State University, Box 871504, Tempe, AZ 85287-1504.

${ }^{3}$ Mount Stromlo and Siding Spring Observatories, Australian National University, Private Bag, Weston Creek ACT 2611, Australia.

${ }^{4}$ Anglo-Australian Observatory, P.O. Box 296, Epping NSW 2121, Australia.

${ }^{5}$ Centre for Astrophysics and Supercomputing, Swinburne University of Technology, Mail \#31, P.O. Box 218, Hawthorn VIC 3127, Australia.

${ }^{6}$ Instituto de Astronomia, Geofisica e Ciências Atomsféricas, Universidade de São Paulo, Rua de Matão, 1226, Cidade Universitária, Sao Paulo, SP, CEP 05508-090, Brazil.

${ }_{7}$ Harvard-Smithsonian Center for Astrophysics, 60 Garden Street, Cambridge, MA 02138 .

${ }^{8}$ Lick Observatory, University of California, 373 Interdisciplinary Sciences, Santa Cruz, CA 95064.
}

Given all that we know about Galactic GCs, it was reasonable to assume that the $\mathrm{GC}$ systems around other spiral galaxies similar to ours would also be similar to ours: generally old GCs having similar ages and metallicities among their constituent stars. However, recently several groups who have studied the M31 GC system have uncovered significant differences between that system and that of our own Galaxy.

Morrison et al. (2004) have shown that one can use accurate radial velocities for M31 GCs to divide them into disk and bulge categories, with the disk GCs having radial velocities that closely follow the disk rotation curve of M31. Beasley et al. (2004) and Barmby et al. (2000) show that M31 has GCs that show A-type spectra, from which one infers that these GCs are very young. Two of us (Y. L. and D. B.) have recently shown that four of the most luminous M31 GCs have far stronger NH features in them than do Galactic GCs measured at otherwise similar, other metal-line indices, such as $\mathrm{CH}$ or $\mathrm{Mg}_{2}$ (Li \& Burstein 2003).

In a follow-up to Li \& Burstein (2003), our group has obtained spectra down to $3250 \AA$ of 22 new M31 GCs spanning a wider range in absolute magnitude than the Hubble Space Telescope (HST) sample used by Ponder et al. (1998). These are combined with six young M31 GCs observed by Barmby et al. (2000) to present a more coherent picture of the M31 GC system. In $\S 2$ of the present paper we give details of the new MMT observations. In $\S 3$ we present the spectra, together with spectra for the Galactic GCs we presented in Li \& Burstein (2003). These spectra convincingly show that M31 contains 
TABLE 1

Basic Information for 22 M31 Globular Clusters

\begin{tabular}{|c|c|c|c|c|c|}
\hline Globular Cluster $^{\mathrm{a}}$ & $V$ Magnitude $^{\mathrm{b}}$ & $\mathrm{Lick} / H S T^{\mathrm{c}}$ & Date of Observation & $\begin{array}{c}\text { Exposure Time } \\
\text { (s) }\end{array}$ & Residual Parameter $^{\mathrm{d}}$ \\
\hline $\mathrm{B} 000-\mathrm{S} 001=$ May II = G1 & 13.7 & $\mathrm{H}, \mathrm{L}$ & 2003 Sep 27/28 & 600 & Bulge \\
\hline B019-S072 = V44 & 14.6 & $\ldots$ & 2003 Sep $27 / 28$ & 1200 & 4.07 \\
\hline B029-S090 = V29. & 16.3 & $\mathrm{~L}$ & 2003 Sep 27/28 & 2700 & -0.32 \\
\hline B158-S213 = V64 & 14.5 & $\mathrm{~L}$ & 2003 Sep 27/28 & 1200 & 1.94 \\
\hline B171-S222 = V87 & 15.0 & $\mathrm{~L}$ & 2003 Sep 26/27 & 1800 & -0.04 \\
\hline В210 & 16.8 & $\ldots$ & 2003 Sep $26 / 27$ & 3600 & 0.11 \\
\hline B216-S267 = V119 & 17.6 & $\ldots$ & 2003 Sep 26/27 & 3600 & -0.10 \\
\hline B218-S272 = V101 & 14,7 & $\mathrm{~L}$ & 2003 Sep 27/28 & 1200 & 0.36 \\
\hline 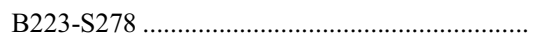 & 15.3 & $\ldots$ & 2003 Sep $26 / 27$ & 1800 & -0.01 \\
\hline 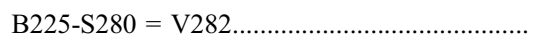 & 14.3 & $\mathrm{H}, \mathrm{L}$ & 2003 Sep $27 / 28$ & 1200 & 1.43 \\
\hline 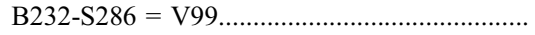 & 15.5 & $\mathrm{~L}$ & 2003 Sep 27/28 & 1800 & 2.52 \\
\hline 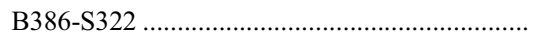 & 15.6 & $\ldots$ & 2003 Sep $26 / 27$ & 2700 & -7.57 \\
\hline 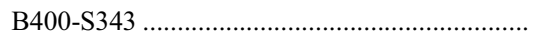 & 16.4 & $\ldots$ & 2003 Sep $26 / 27$ & 3600 & -8.47 \\
\hline 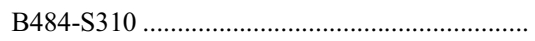 & 18.6 & $\ldots$ & 2003 Sep $27 / 28$ & 4500 & 0.59 \\
\hline 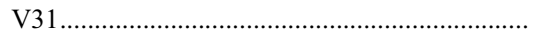 & 17.0 & $\ldots$ & 2003 Sep 26/27 & 3600 & 0.02 \\
\hline
\end{tabular}

${ }^{a}$ The first designations conform to the Barmby et al. (2000) M31 GC designations; May II = Mayall II, which is also called G1 in some papers; V = Vetesnik number.

${ }^{\mathrm{b}}$ The observed $V$ magnitude of the cluster, taken from either the Sargent et al. (1977) list (as given in Burstein et al. 1984) or the Barmby et al. (2000) list.

${ }^{\mathrm{c}} \mathrm{L}=$ also observed by Burstein et al. (1984); $\mathrm{H}=$ also observed by Ponder et al. (1998).

${ }^{\mathrm{d}}$ The residual parameter of Morrison et al. (2004); a value less than \pm 2.2 makes it possible that this is a disk GC in M31. However, not all M31 GCs with such residual parameters are disk GCs, as is evident in our own data.

GCs with a range of age from as young as $\sim 100$ Myr to $5 \mathrm{Gyr}$ old, to as old as the oldest Galactic GCs. They also clearly show the differences in nitrogen abundance between the old M31 GCs and the old Galactic GCs. In $\S 4$ we discuss the various implications of the differences found between the M31 and Milky Way GC systems for galaxy formation and the existence of young GCs in spiral galaxies and tidally interacting galaxies. In $\S 5$ we give our summary.

\section{THE MMT OBSERVATIONS}

Li \& Burstein (2003) were assigned two nights with the $6.5 \mathrm{~m}$ MMT on 2003 September 26/27 and 27/28 to use the Blue Channel spectrograph to obtain spectra that reach to the blue atmospheric limit of a selection of M31 GCs. The GCs chosen for this study were taken from two sources. First, it was desirous to reobserve many of the M31 GCs observed by Burstein et al. (1984) in order to ensure that we were on the same absorptionline system as defined by the Lick Observatory data. Many of these are also among the brightest M31 GCs. For all but one of these Lick Observatory-observed M31 GCs, Morrison et al. (2004) have defined whether or not these systems follow closely the rotation curve of M31 or not.

Second, our remaining GC sample is taken randomly from among the GCs that have the "residual" parameter defined by Morrison et al. (2004). It is this residual parameter that Morrison et al. (2004) use to measure how close or how far the radial velocity of a GC in M31 is to the rotation curve of that galaxy. However, one can only be sure if a GC is a bulge GC, as there are no disk GCs with a residual parameter greater than 2.2 (e.g., as can be seen in Fig. 6 of Morrison et al. 2004). Hence, while one can choose GCs that are clearly bulge GCs, choosing disk GCs is more problematic. Given the distribution of residuals for disk GCs in Figure 6 of Morrison et al. (2004), we chose those M31 GCs with residual parameters close to zero to maximize our chances of picking up true disk GCs in M31.

We employed the 3001 blue grating with the Blue Channel of the MMT spectrograph blazed at $4800 \AA$ with a $2^{\prime \prime} \times 180^{\prime \prime}$ long slit (the $2^{\prime \prime}$ width to cover most of the area of these spatially resolved GCs, the length to provide the background for many of these GCs, most of which was due to M31 itself). This combination yielded a spectral resolution close to $13 \AA$. A grating angle of 1.12 was sufficient to get us to $3200 \AA$ in the blue but still gives us up to $6000 \AA$ in the red, redward of which we run into second-order confusion with the spectra. Standardstar observations of $\mathrm{BD}+33^{\circ} 2642, \mathrm{G} 138-31, \mathrm{BD}+28^{\circ} 4211$, G191-B2B, and Feige 110 are interspersed with the program objects, to provide calibrations for spectral energy distributions (SEDs) for these GCs. Table 1 gives a log of our observations, including the GCs observed, giving their Battistini et al. (B) and Sargent et al. (S) numbers, as well as their Vetesnik numbers (when available; Battistini et al. 1980; Sargent et al. 1977; Vetesnik 1962a, 1962b); if there are HST (H) or Burstein et al. (L) observations of these clusters; the exposure times used for their spectra; their apparent magnitudes (not corrected for Galactic extinction); and their Morrison et al. (2004) residual parameters. We adopt a value for $V$ magnitude Galactic extinction for these M31 GCs from Burstein et al. (1984) of $A_{V}=0.25 \mathrm{mag}$.

\section{THE SPECTRA}

As seen in Table 1 and stated above, 11 of the M31 GCs in our sample were nominally observed by Burstein et al. (1984). 


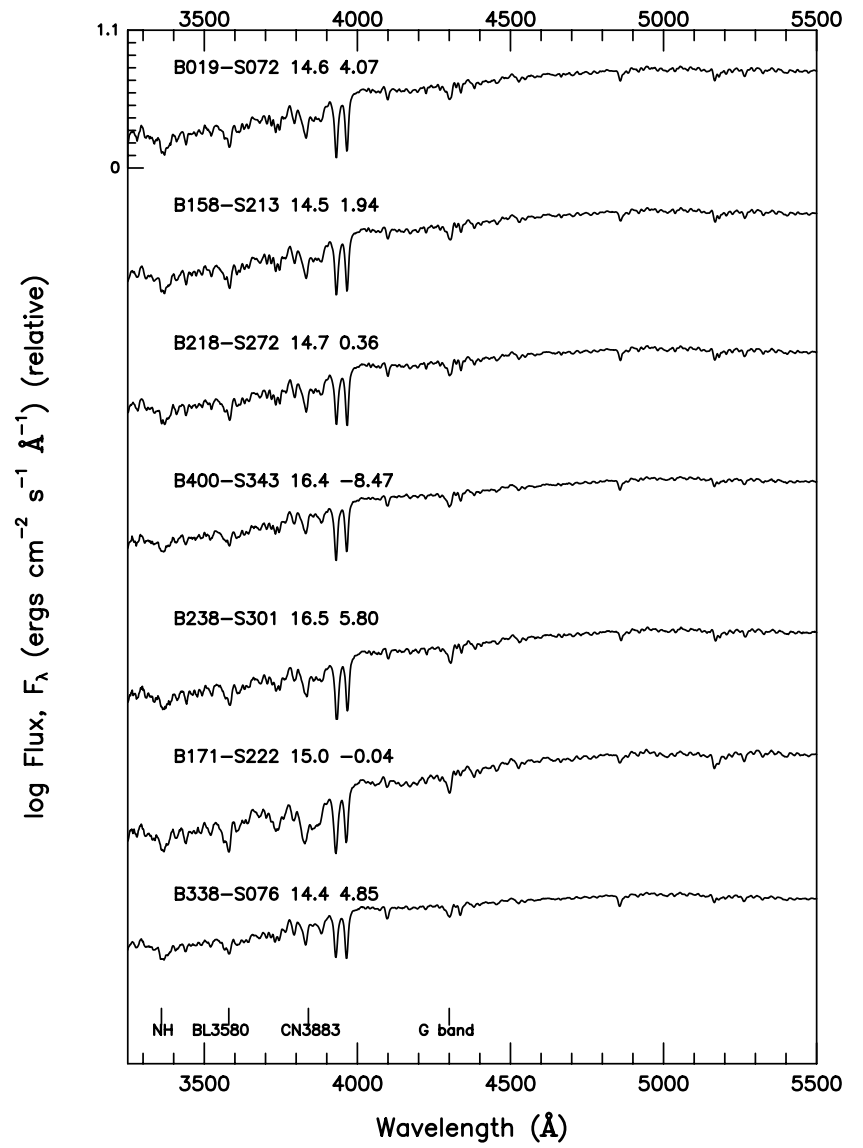

FIG. 1.-Spectra for 7 of the 14 M31 GCs observed with the MMT that are both as old as the oldest Galactic GCs and have strong $\mathrm{NH}$ in their spectra. Their $V$ magnitudes and Morrison et al. (2004) residual parameters are given by their names. The fluxes for these spectra are plotted on a $1.1 \mathrm{dex} \log$ scale.

However, as we see when we compare line strengths to the Lick Observatory M31 GC sample, there is a question about the identity of one of the Lick Observatory M31 GCs. Figures 1-4 contain the spectra we obtained for these 22 M31 GCs. Both their apparent $V$ magnitude and their Morrison et al. (2004) residual parameter are given for each GC in Figures $1-4$ (when available). Figure 5 gives the spectra that we published in Li \& Burstein (2003) for eight Galactic GCs for comparison. Figure 6 gives the Keck spectra from Barmby et al. (2000) for six of the GCs that they indicated as being "young?" in their table. Only two of the Barmby et al. (2000) GCs shown in Figure 6 have Morrison et al. (2004) residual parameters, and both of these are consistent with these being disk GCs. The Keck spectra have been "Hanning smoothed" [i.e., a running average of flux of $\left(0.5 f_{1}+f_{2}+0.5 f_{3}\right) / 2$ ]. (Note that the Barmby et al. 2000 Keck spectra do not go much below $3700 \AA$ A.) All spectra in Figures 1-6 are displayed in terms of $\log$ (flux) versus wavelength. The log stretch used for all of the spectra in this paper is $1.1 \mathrm{dex}$ (vs. 0.7 dex used in $\mathrm{Li} \&$ Burstein 2003).

Three things are notable about these new M31 GC spectra:

1. For the oldest 12 of the new M31 GCs (two are the same as previously observed by HST; see Table 1), it is obvious that the strong NH absorption we saw for the spectra of four M31 GCs in Ponder et al. (1998) is also present in these new M31 GC spectra. However, there is only one M31 GC with a $V$ magnitude fainter than 17 in this group (B001-S039), and its spectrum is far noisier than the other spectra in this group. Otherwise, all

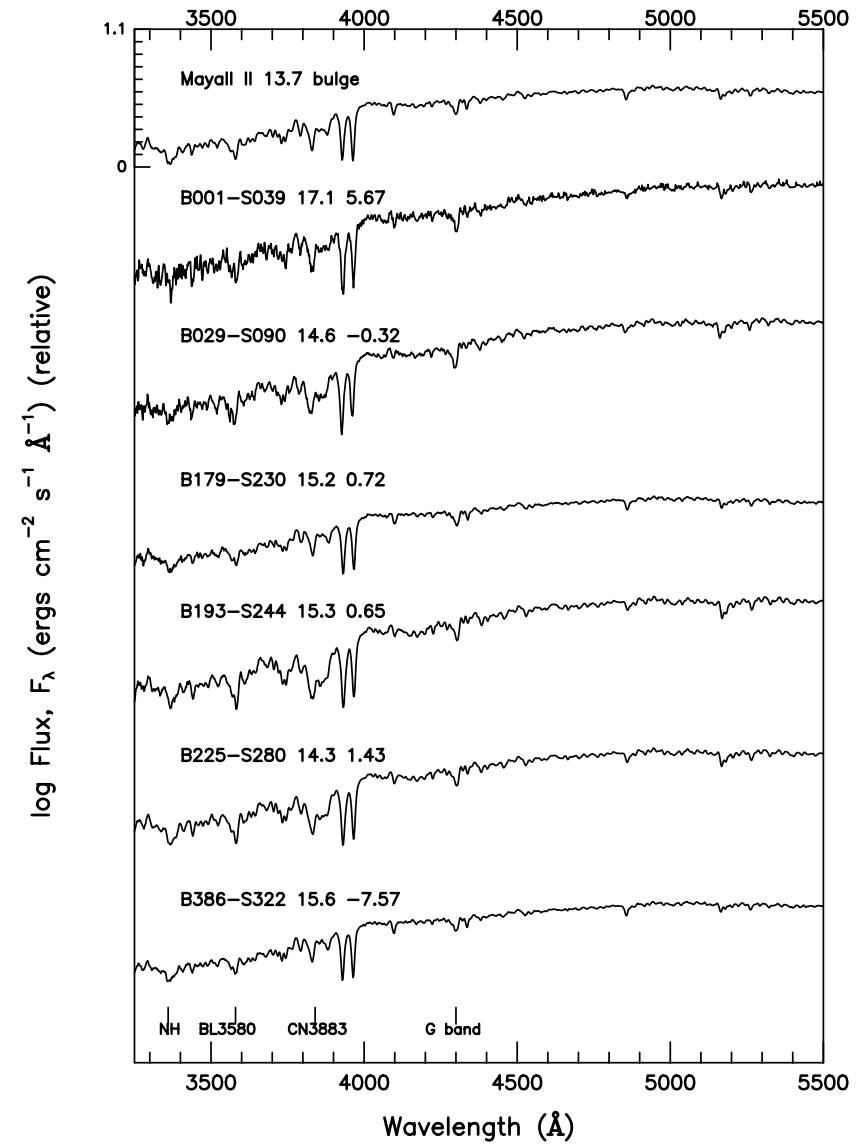

FIG. 2.- Spectra for the seven other old M31 GCs, plotted on the same log flux scale as in Fig. 1.

of the GCs with strong $\mathrm{NH}$ in their spectra have $V$ magnitudes between 13.7 and 16.5 (or, given a distance modulus to M31 of 24.38 [Freedman et al. 2001], absolute $V$ magnitudes from near -11 to -8 , once Galactic extinction is taken into account). Most of the 14 GCs in Figures 1 and 2 are bulge/halo clusters, according to either their position on the sky (Mayall II) or their Morrison et al. (2004) residual parameter. Several have disklike Morrison et al. (2004) residuals but have spectra like those of the other bulge GCs (and, hence, are put in Figs. 1 and 2).

2. In contrast, six of the M31 GCs we observed have Morrison et al. (2004) residual parameters that place them squarely within the M31 disk system. All six have quite remarkable spectra (Fig. 3). All show a very strong Balmer decrement, a strong Balmer line series with the broad absorption hydrogen lines typical of dwarf A stars, and $\mathrm{Ca}$ II $\mathrm{H}+\mathrm{H} \epsilon$ much stronger than the $\mathrm{Ca}$ II $\mathrm{K}$ line. Of these six M31 GCs, two are in common with the Barmby et al. (2000) published sample of M31 GCs (B216-S267 and B315-S038). The spectra of six GCs taken from the sample observed by Barmby et al. (2000; Fig. 6) show similar A-type stellar behavior, with a wider range of $\mathrm{Ca}$ II $\mathrm{H}$ and $\mathrm{K}$ issues than those observed with the MMT. If we include all of the M31 GCs indicated as "young?" by Barmby et al. (2000), this makes as many as 19 young GCs in M31 known so far. (However, not all of the Barmby et al. 2000 "young?" GCs have Morrison et al. 2004 parameters, so it is not clear if only disk GCs are preferentially young GCs.)

We can estimate how young these M31 GCs are by using the published integrated spectra of the young GCs in the Large Magellanic Cloud (LMC) given by Leonardi \& Rose (2003). As the LMC spectra are also fluxed, we see that the youngest 


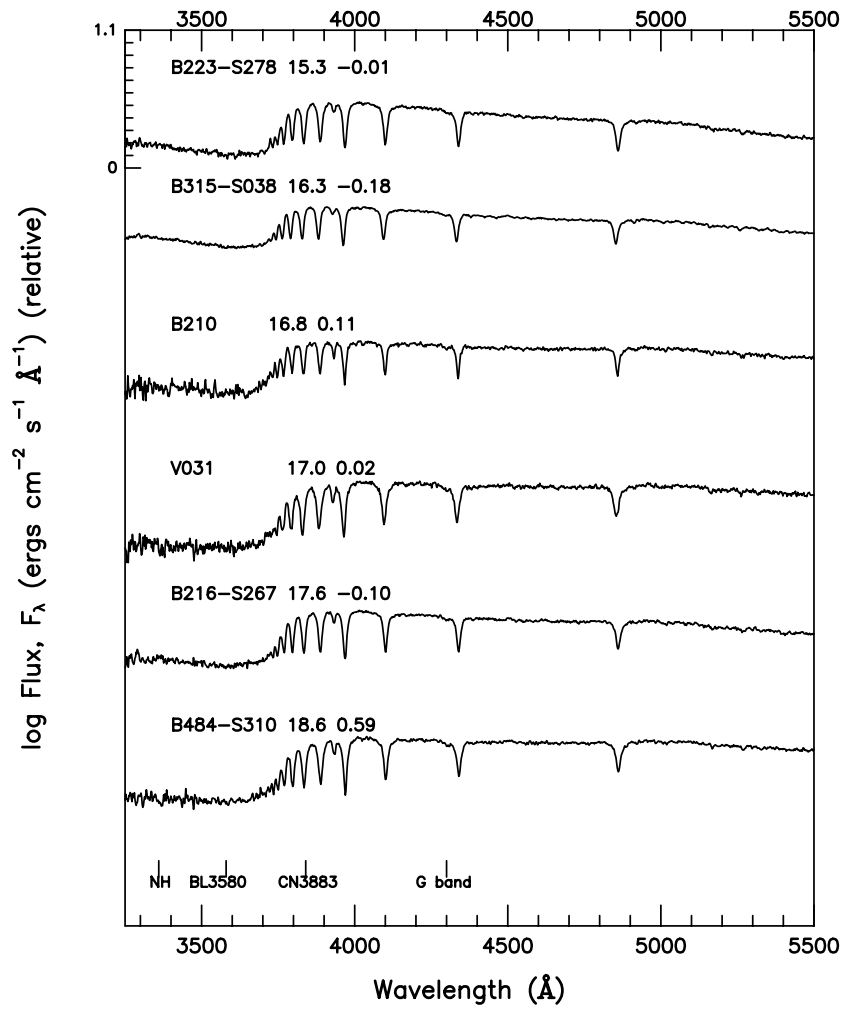

FIG. 3.-Spectra for the six 500 Myr old M31 GCs, plotted on the same log flux scale as in Fig. 1. Note the strong Balmer jump for these GCs, as well as the strong Balmer line series in their spectra. The log flux scale used is the same as in Fig. 1.

LMC clusters (ages 200 Myr or younger) have spectra that have deeper Balmer lines than the young M31 GC in Figure 3. Hence, an estimate of $500 \mathrm{Myr}$ is not an unreasonable estimate for the ages of these six MMT M31 GCs. It is possible that some of the Barmby et al. (2000) clusters are somewhat younger than this age (e.g., B380-S313 and B321-S046) and one perhaps as old as 1 Gyr (B347-S154). While Barmby et al. (2000) point out that others in the past have indicated that M31 contains such young GCs, it really takes these kind of spectra to bring this issue home to all of us.

3. The two M31 GCs in Figure 4 (B232-S286 and B311S033) also seem younger than the oldest M31 GCs. Their spectra appear similar (modulo signal-to-noise ratio $[\mathrm{S} / \mathrm{N}]$ issues) to the spectra of the LMC clusters NGC 1795 and NGC 1754 as seen in Leonardi \& Rose (2003), which have estimated ages of order 5 Gyr.

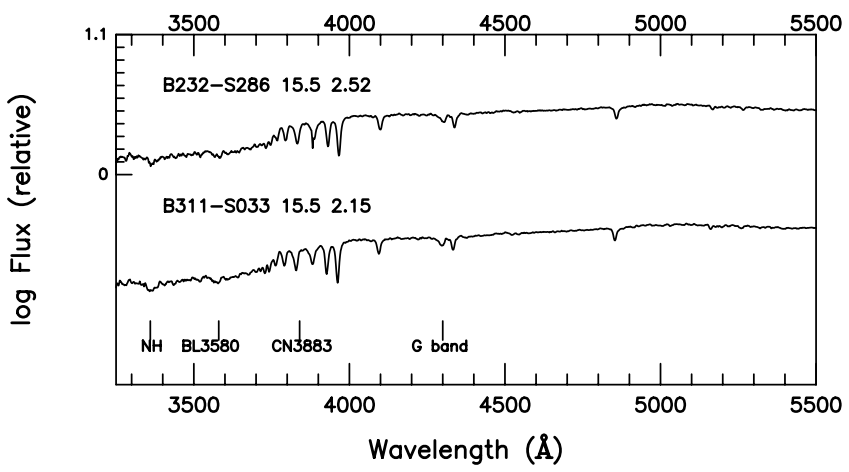

FIG. 4.- Spectra for the two 5 Gyr old GCs found in our M31 MMT data, plotted on the same log flux scale as in Fig. 1.

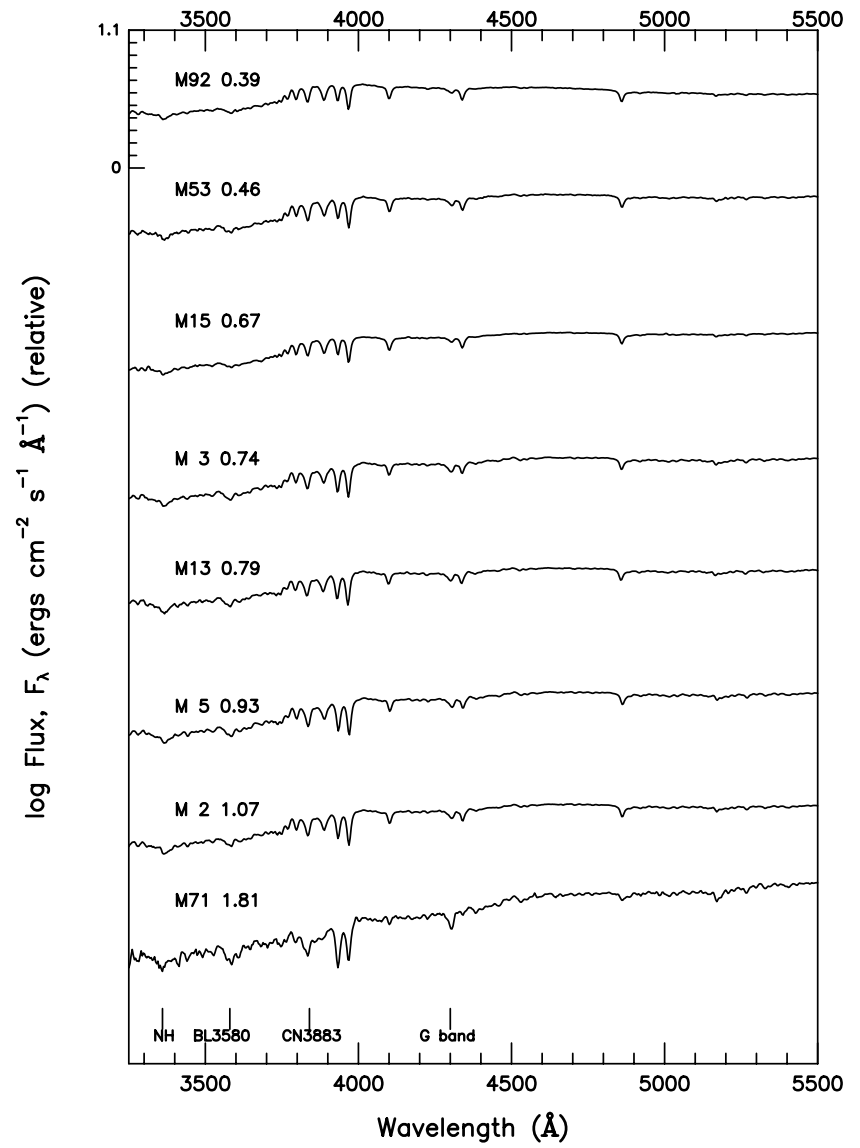

FIG. 5.- Spectra for the eight Milky Way GCs from Li \& Burstein (2003) shown at the same plotting scale as Figs. 1-4 for comparison. The number by the name of each Milky Way GC is the value for the Lick index $\langle\mathrm{Fe}\rangle$ for this GC.

We can compare the absolute magnitudes of these young M31 GCs with their compatriots in the LMC. Using a distance modulus of 18.50 to the LMC (e.g., Freedman et al. 2001), the LMC GCs that have ages $200 \mathrm{Myr}$ and younger have absolute $B$ magnitudes from $M_{B}=-6.5$ to -9.0 , reddening corrected. The two older, $\sim 5$ Gyr old LMC GCs have absolute magnitudes of -6.5 and -7.3 .

There is no evidence from the fluxed spectra shown in Figures 1-4 that any of the M31 GCs we observed have significant reddening from inside M31 itself, so we use the Galactic estimate of $A_{V}=0.25 \mathrm{mag}$ for all of the clusters. With this value of extinction, the younger M31 GCs in Figures 3 and 6 have apparent $V$ magnitudes $15.3-18.6$, or absolute magnitudes from -9 to -5.5 . This is quite similar to the range of absolute magnitudes of the 200 Myr LMC GCs. The absolute $B$ magnitudes of the two $\sim 5$ Gyr GCs are both close to -9 . From this, we think we can conclude that the younger M31 GCs are quite analogous to the younger LMC GCs, in that their high luminosities are not necessarily indicative of their overall mass. In contrast, the absolute magnitudes of the two 5 Gyr old M31 GCs indicate that they are considerably more massive than their LMC counterparts.

\subsection{The Line-Strength Measures}

Among the absorption-line systems we have measured in our MMT spectra, for the present paper we include those of $\mathrm{NH}, \mathrm{CH}$ (the $\mathrm{G}$ band), and $\mathrm{Mg}_{2} .{ }^{9}$ Measurements of the latter

\footnotetext{
${ }^{9}$ Quantitative measurement of all of the absorption-line systems in our spectra will be published in the Ph.D. thesis of Y. L.
} 


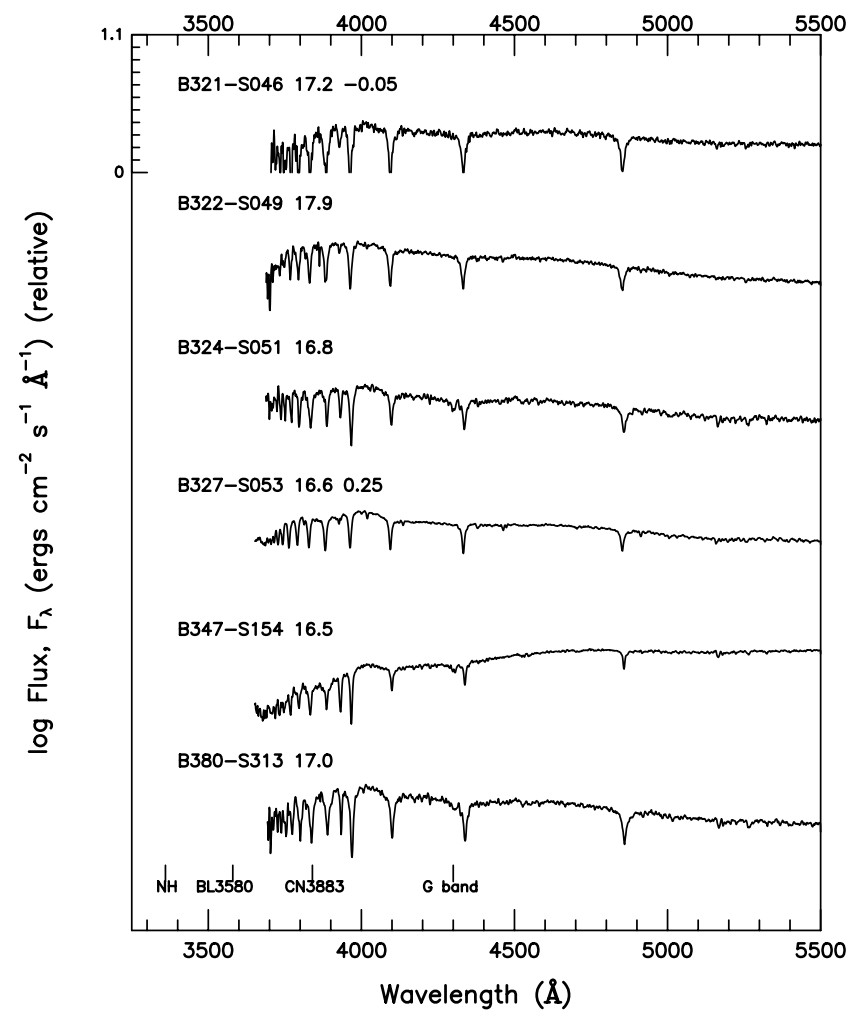

FIG. 6.-Keck spectra of Barmby et al. (2000) for six of the "young?" M31 GCs that they so indicated in their summary table. These spectra have been Hanning smoothed to remove much of the apparent noise in the original data. Note that these spectra do not extend much blueward of $3700 \AA$.

two indices are defined on the Lick Observatory system (e.g., Burstein et al. 1984), but with the caveat that our fluxed spectra define a different long-wavelength continuum than do the quartz-calibrated spectra that define the Lick system. We define the NH parameters according to the precepts of Davidge \& Clark (1994), who did use fluxed data to obtain their measurements. Further, given that we separately observed the Galactic GCs by scanning the telescope in a large swath over them, we also need to see if the transformation to the Lick system is different for the Galactic GC as opposed to our MMT spectra for the M31 GC. We give both our line-strength data and those from the Lick observations (taken from Trager et al. 1998, for consistency) in Table 2.

We note that one of the M31 GCs in Table 2 shows a marked difference in the $\mathrm{Mg}_{2}$ and $\mathrm{CH}$ measures we obtained for it compared to what was published in 1984: B232-S288/Vetesnik 99. Our spectra clearly show this cluster to be very metal-poor, while the Lick spectra clearly show it to be more metal-rich. A check of our observing log position for this cluster confirms that we observed the cluster we have so identified. Such confirmation is not available for the older Lick data, so we assume that the M31 cluster listed as V99 in the Lick paper is not V99. Hence, we exclude this cluster from the line-strength comparisons with the Lick Observatory data (but not from other analyses).

For the eight Galactic GCs, we find that the difference in magnitude for $\mathrm{Mg}_{2}$, in the sense of Trager et al. (1998) minus us, is $-0.008 \pm 0.003$ average difference, with 0.008 mag error per object. Essentially all of the error is observational. For the 10 remaining M31 GCs that are in common with the Trager et al. (1998) data, we have $+0.029 \pm 0.003$ mag average difference, or $0.010 \mathrm{mag}$ error per observation. Most of the error is a combination of observational errors from both data sets.

In the case of $\mathrm{CH}$ we find an average difference for the eight Galactic GCs of $0.006 \AA$ (Trager et al. 1998 minus us), with a mean error of $0.171 \AA$ and a single observational error of $0.484 \AA$. We note that the mean error of the Lick observations is $0.36 \AA$, more than sufficient to account for the errors observed between these two sets of data. For the 10 M31 GCs, we get an average difference of $0.146 \AA$, with a mean error of $0.235 \AA$ and a single observational error of $0.742 \AA$. Given that the mean difference is much smaller than the mean errors for the $\mathrm{CH}$ measurements of both the Galactic GCs and the M31 GCs, we assume that we are on the Lick Observatory line-strength system for $\mathrm{CH}$ for both sets of data.

We note that to place the $2.4 \mathrm{~m}$ Bok telescope observations of the 125 Lick Observatory stars observed by Y. L. for his $\mathrm{Ph}$.D. thesis, offsets in the sense of Trager et al. (1998) minus Y. L. are $+0.006 \pm 0.001$ for $\mathrm{Mg}_{2}$ and $+0.211 \pm 0.04$ for $\mathrm{CH}$. These differences have been applied to the stellar data used in our figures. The differences in getting to the standard Lick system for stars versus GCs are understandable in terms of both $\mathrm{Mg}_{2}$ and $\mathrm{CH}$, given the wide wavelength regions that define both indices and the differences in SEDs between individual stars and integrated stellar populations.

In Table 2 we list the absorption-line values for $\mathrm{NH}, \mathrm{CH}$, and $\mathrm{Mg}_{2}$ for both the M31 GCs we observed here and the Galactic GCs we presented in Li \& Burstein (2003), together with their errors and the absolute magnitudes of these clusters. In Figure 7 we present the relationships among $\mathrm{NH}, \mathrm{CH}$, and $\mathrm{Mg}_{2}$ for the 17 older M31 GCs (including the two 5 Gyr old GCs, but not the $500 \mathrm{Myr}$ old GCs), including the two HST-observed M31 GCs not observed by us with the MMT, as well as for the Galactic GCs. The M31 GCs observed with the MMT and the Galactic GCs have their $\mathrm{Mg}_{2}$ corrected by adding 0.029 and $-0.008 \mathrm{mag}$, respectively, to their measured values to bring them into accord with the Lick standard system. (We note that, given the scale at which Fig. 7 is plotted for $\mathrm{CH}$, the mean offset calculated for $\mathrm{CH}$ for the M31 GCs is smaller than the sizes of the symbols used to represent these GCs.)

As one can see, the new M31 GCs that are the oldest have the same kind of enhanced $\mathrm{NH}$ absorption feature as do the original four M31 GCs that we investigated with HST FOS. It is only the two 5 Gyr old M31 GCs that have NH indices similar to those of Galactic GCs. Thus, we find three differences among the GCs of M31 and those of our own Milky Way:

1. M31 contains GCs with a range of age from $\sim 100 \mathrm{Myr}$ old to 500 Myr old to 5 Gyr old to as old as the oldest Galactic GCs. In other words, M31 contains GCs that are much younger than the known GCs in our own Galaxy.

2. While both the oldest and most metal-poor M31 and Galactic GCs show enhanced nitrogen abundances in their integrated spectra, the main-sequence stars in the M31 GCs have markedly more nitrogen in their atmospheres than do the main-sequence stars in either the Milky Way GCs or most field stars in the Milky Way that are of similar $\mathrm{CH}$ or $\mathrm{Mg}_{2}$ line strengths.

3. As Morrison et al. (2004) have shown, M31 has a set of GCs that closely follow the rotation curve of M31. None of the known GCs in the Milky Way do this.

\subsection{Keeping Score with the Predictions}

One of the predictions of Li \& Burstein (2003) from their $\mathrm{GC}$ and halo formation scenario is that younger GCs in other 
TABLE 2

The Data for 22 M31 and 8 Galactic Globular Clusters

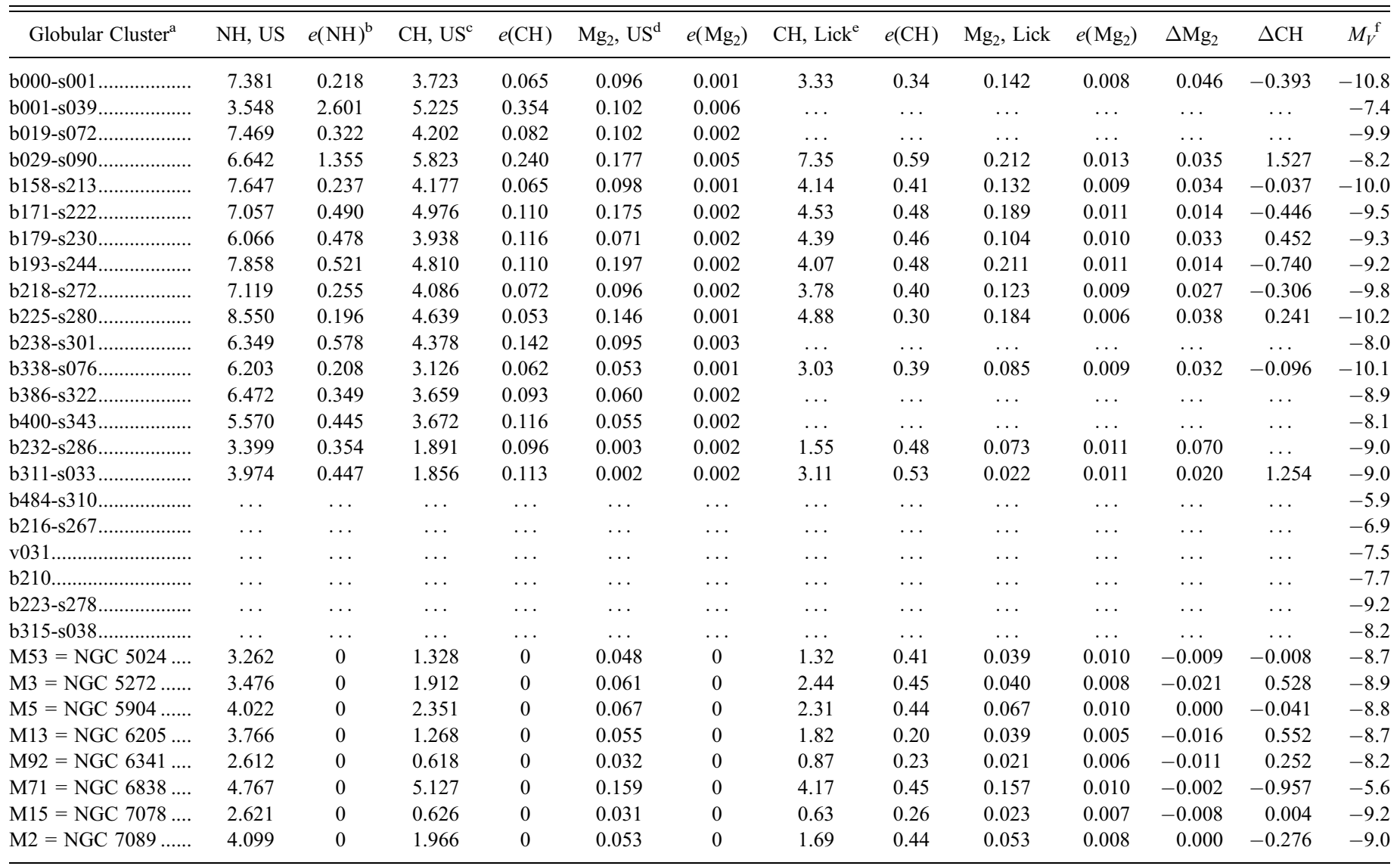

${ }^{\text {a }}$ The GCs are listed in the following way: the first 14 are the old, NH-rich M31 GCs, the next 2 are the 5 Gyr old GCs, the next 6 are the 500 Myr old GCs, and the final 8 are the Galactic GCs. No absorption-line strengths are given for the $500 \mathrm{Myr}$ old M31 GCs. M31 GC names are as in Table 1. Galactic GC names are given as both Messier and NGC numbers.

${ }^{\mathrm{b}} \mathrm{NH}$ equivalent widths are measured in angstroms.

${ }^{\mathrm{c}} \mathrm{CH}$ equivalent widths are measured in angstroms.

${ }^{\mathrm{d}} \mathrm{Mg}_{2}$ values are measured in magnitudes.

e No Lick data are given if not observed by Burstein et al. (1984).

${ }^{\mathrm{f}}$ Absolute $V$ magnitude, extinction corrected using the extinction values given in Harris (1996), and a value of $E(B-V)=0.08$ for M31 GCs.

galaxies should have $\mathrm{NH}$ in proportion to their normal metallicity, as compared to the enhanced NH we see in the oldest clusters. In this regard, we note that the two 5 Gyr old GCs in M31 do have NH much weaker than their $10 \mathrm{Gyr}$ old GC cousins, consistent with this prediction. Note, however, that even though the 5 Gyr old M31 GCs are weaker in $\mathrm{NH}$ than their older cousins, they are of comparable $\mathrm{NH}$ strength relative to the $\mathrm{NH}$ measures for the old Galactic GCs. This is perhaps an indication that nitrogen is greatly enhanced in M31 GCs in general, not just in their oldest GCs. This makes one wonder whether older field stars in M31 have enhanced nitrogen abundances relative to Galactic field stars.

Separately, as pointed out by Tomkin \& Lambert (1984), $\mathrm{CH}$ (the $\mathrm{G}$ band) and $\mathrm{NH}$ have very similar dissociation energies. The fact that $\mathrm{CH}$ lies $1000 \AA$ to the red of $\mathrm{NH}$ implies that the weakness of $\mathrm{CH}$ in the integrated spectra of the M31 GCs (and Galactic GCs) relative to the Galactic stars (see Fig. 7) means that carbon is very underabundant relative to nitrogen in these GCs (and also in the Galactic GCs). Such was also found for main-sequence stars in several Galactic GCs (e.g., Briley et al. 2004). If these abundance differences are truly primordial in origin, are not these abundance anomalies telling us something about the elements the first stars produced?

\section{THE HIERARCHICAL-CLUSTERING-MERGING PARADIGM AND ITS IMPLICATION FOR GLOBULAR CLUSTERS OF OTHER GALAXIES}

The hierarchical-clustering-merging (HCM) paradigm is the current view of galaxy formation (e.g., Burstein et al. 1997; Kauffmann et al. 1997). HCM pictures galaxy formation as small galaxies combining to form larger galaxies. In an HCMdominated universe, each galaxy will undergo a series of major and minor mergers in its lifetime. If the merger process does not produce an E or S0 galaxy, then it will produce a spiral galaxy. Since there are more spiral galaxies than E/S0 galaxies in the universe (e.g., the galaxies in both the Uppsala General Catalog [Nilson 1973] and the Third Reference Catalog of Bright Galaxies [de Vaucouleurs et al. 1991] are dominated by spiral galaxies), it is clear that if HCM is the dominant mode of galaxy formation, spiral galaxies are its preferred output. In sum, the current evidence is consistent with the hypothesis that the HCM paradigm applies to all giant, E, S0, and spiral galaxies (e.g., Burstein et al. 1997). 

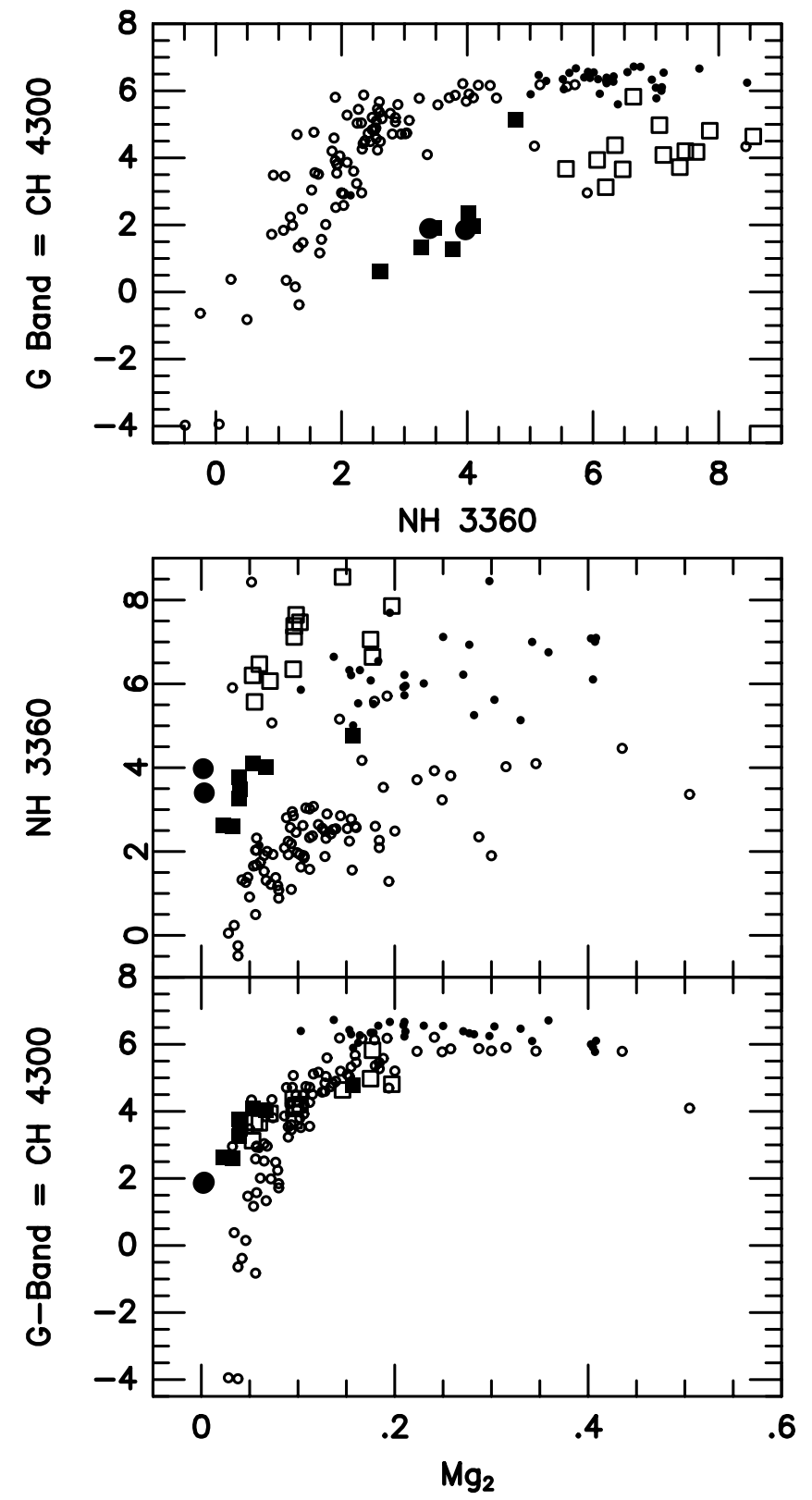

FIG. 7.-Top: $\mathrm{CH} 4300$ (the G band), plotted in units of angstroms, vs. NH, in units of angstroms, for the dwarf stars of Yong Li's Ph.D. thesis (small open diamonds), the giant stars in his thesis (small filled circles), the Galactic GCs (large filled squares), 15 of the older MMT M31 GCs (large open squares; M31 GC B001-S039 is excluded as its NH measure is too noisy), and the two 5 Gyr old M31 GCs (large filled circles). Bottom: $\mathrm{NH}$ and $\mathrm{CH}$ (the $\mathrm{G}$ band), in units of angstroms, plotted vs. $\mathrm{Mg}_{2}$ in units of magnitude. Note the lack of difference in $\mathrm{CH}$ vs. $\mathrm{Mg}_{2}$ for the old GCs vs. Galactic stars, while in $\mathrm{NH}$ vs. $\mathrm{Mg}_{2}$, both M31 and Galactic GCs stand out vs. the stars. Only the two $5 \mathrm{Gyr}$ old M31 GCs stand out in the $\mathrm{CH}$ vs. $\mathrm{Mg}_{2}$ diagram. All error bars for the GCs are of comparable sizes to the plotted points.

This then begs a series of questions that follow in logical steps: (1) How does the HCM paradigm relate to young M31 GCs that we find in M31 but do not find in our own Galaxy? (2) Where else do we find young GCs in the Local Group? (3) Where do we find most of the dwarf systems in the Local Group? (4) Why do young GCs preferentially form in irregular or small spiral galaxies? (5) Where do the young GCs seen in tidally interacting galaxies come from?

The second question is the most obvious to answer. We see young GCs in the irregular companion galaxies to the Milky
Way, the Large and Small Magellanic Clouds, as well as likely in the low-luminosity Sc galaxy in the Local Group, M33 (e.g., Ma et al. 2001). Indeed, we have used the integrated SEDs of LMC young GCs obtained by Leonardi \& Rose (2003) to estimate the ages of our younger M31 GCs.

The answer to the third question comes from the fact that $98 \%$ of the mass and luminosity of galaxies in the Local Group is contained in just M31 and the Milky Way. If you plot the known positions of the dwarf galaxies in the Local Group (data kindly given to D. B. by Eva Grebel), one sees that both M31 and the Milky Way have close contingents of these dwarf galaxies (e.g., the LMC, SMC, and various dEs and dSphs close to the Milky Way).

Put the answers together to the previous two questions and we have our answer to the first question. The young GCs seen in M31 likely came from the small spiral and/or irregular galaxies that once were companions of M31 and have since merged with M31. Since the LMC is less than 1\% the mass of M31, mergers with LMC-like irregular galaxies would do little to the disk of this galaxy. That such a merger history has taken place in M31 is consistent with what Brown et al. (2003) have found in their deep HST investigation of the halo of M31. Namely, they find an intermediate-aged stellar population, which could be the remnants of the dwarf system that produced the 5 Gyr old GCs in M31. Furthermore, the fact that one sees what has been interpreted as a severe warp in front on the bulge of M31 (Bajaja \& Shane 1982; Brinks \& Burton 1984) now can also be interpreted as the wraparound debris from merger remnants.

If a galaxy similar to the LMC were accreted by M31 during the past 100-200 Myr, then all of the GCs in that irregular galaxy would now become GCs of M31, would they not? Furthermore, if that accretion took place such that the irregular galaxy would be tidally disrupted along the disk of M31, most of the GCs in this irregular galaxy would assume the rotation velocity of M31, forming its thin disk of GCs. It is in this thin disk of GCs that we find the young GCs of M31. (However, as noted above, these may not be the only types of disk GCs in M31.)

If this is true for M31, why is it not true for the Milky Way? Because, in the HCM paradigm, what the merger history is of one galaxy does not predict what the merger history of another galaxy will be, even if they are close neighbors! Evidently, the Milky Way has not had a merger of an irregular galaxy like the LMC or the SMC in the past 10 billion years or so, or else we would see younger GCs in our own Galaxy.

Is it not likely that many, if not most, of the GCs in spiral galaxies were once in the smaller systems that combined to make the spiral galaxies we see today? If this is true, it is also true that the HCM paradigm does not dictate how and when such accretion will take place. As such, we feel that it is a prediction of the HCM paradigm that the GC systems of all spiral galaxies (and, for that matter, also gE and S0 galaxies) are assembled in a rather haphazard "big-fish-eats-small-fish" manner. We see this happening today in our own Galaxy with the Sagittarius dwarf galaxy (e.g., Ibata et al. 1994) and the Canis Major dwarf (Forbes et al. 2004), both of which we are accreting, each adding four to six GCs to our GC contingent (we note that two of the GCs the Milky Way is accreting from each dwarf galaxy have ages $~ 7$ Gyr; e.g., Forbes et al. 2004).

Why do small spiral and irregular galaxies preferentially form young GCs? We think that this is because in such galaxies one does not have substantial rotational shear. This permits large molecular clouds (e.g., with masses up to $10^{9} M_{\odot}$; Harris $\&$ Pudritz 1994) to form that will not be sheared into smaller 
systems. In the Milky Way such sheared stellar systems tend to form the open clusters we find in our disk. If this interpretation is correct, then the fact that M31 has both relatively young GCs ( $\sim 500 \mathrm{Myr})$ and moderately old GCs ( 5 Gyr) strongly suggests that M31 went at least a series of separate merging events with its contingent of small companion galaxies that contained many more GCs than the two dwarf galaxies that the Milky Way is currently absorbing (e.g., the Sagittarius dwarf galaxy and the Canis Major dwarf; Ibata et al. 1994; Forbes et al. 2004).

If our interpretation of the origin of the young GCs in M31 is correct, then it shows that we might be able to study the relatively recent (say, the last 10 Gyr or so) merger history of other spiral galaxies by measuring the ages of their GCs. This possibility needs to be tested.

If young GCs are preferentially formed in small galaxies with little or no net rotation velocities, then where do the young GCs that have been found around the Antennae galaxies (e.g., Whitmore \& Schweizer 1995) come from? As an alternative hypothesis to these young clusters having been formed during the tidal interaction, what is the chance that either of the Antennae galaxies (NGC 4038/4039) had one or two irregular galaxies containing young GCs that were "brought along for the ride?" Until we know more about where and how young GCs are formed outside of the Local Group, this idea is consistent with what we see for tidally interacting galaxies.

The difference between M31 and Milky Way GCs that we cannot explain is why the GCs in M31 have markedly stronger $\mathrm{NH}$ absorption than do Galactic GCs of similar metallicities. To date, the M31 GCs for which we have found very strong NH absorptions have luminosities ranging from - 11 (Mayall II) to -8.5 to -9 (with apparent $V$ magnitudes of $15.5-16.0$ ). Ironically, all of the less luminous M31 GCs we took with the MMT are of the young kind.

However, even if this is an issue of the luminosities of the oldest GCs in spiral galaxies, this still begs the question of how one gets a wide range of metallicity among these luminous GCs that spans a similar range as among the Galactic GCs. Of the three known differences among the GC systems of M31 and the Galaxy, it is the difference in nitrogen abundance in them that is still the most puzzling and opens up a number of questions that need answers.

\section{SUMMARY}

In this paper we present evidence that the GC systems of the Milky Way and M31, the two large spiral galaxies in the Local Group, have very different evolutionary histories. Whereas the Milky Way GCs are uniformly very old (10-12 Gyr), those of M31 evidence at least three separate age epochs: 100-500 Myr,
5 Gyr, and 10-12 Gyr old. We strongly suspect that the younger GCs in M31 came from mergers of M31 with its associated irregular galaxies in the past. We note that it might be happenstance that the Milky Way has not yet absorbed the Magellanic Clouds, for if it did, our Galaxy would also have a large number of young GCs in it.

We now find that there are at least three clear differences between the GCs in M31 and those in the Milky Way:

1. M31 contains GCs with a wide range in age, while the Milky Way does not.

2. While both Milky Way and M31 GCs show enhanced nitrogen abundances, the nitrogen abundance of the M31 GCs is clearly greatly enhanced relative to that seen in the Milky Way GCs. Why this is the case is still unknown. Is it possible that nitrogen is overabundant in M31 as a system, as opposed to just in its old GCs?

3. Morrison et al. (2004) find that M31 contains a subset of GCs whose radial velocities closely follow the disk rotation curve of M31. All of the 500 Myr old GCs we and Barmby et al. (2000) find in M31 that have Morrison et al. (2004) residual parameters (about half) are part of these disk clusters. No such GC component of the Milky Way has yet been discovered.

In contrast to what we see for $\mathrm{NH}, \mathrm{CH}$ (of similar dissociation energy as NH; see Tomkin \& Lambert 1984) is not enhanced in the GCs amond the GCs of either galaxy relative to Galactic stars, save for the two 5 Gyr old GCs in M31.

From this evidence, it is clear that if galaxies are formed via the HCM paradigm, then it is likely the case that each spiral galaxy has its own contingent of GCs acquired from its contingent of dwarf systems, which, in principle, can span a wide range in age. Furthermore, it also pegs the Milky Way at one end of this spectrum, in that almost all of the GCs in the Milky Way are old, implying that the vast majority of such mergers occurred more than 10 Gyr ago for our Galaxy. As such, we have a very biased view of GC formation history in spiral galaxies through the study of the GCs in our own Galaxy. It will only be by more thorough spectroscopic investigation of the GCs in spiral (and also elliptical?) galaxies outside the Local Group that the full extent of what the HCM paradigm means for the GC systems of galaxies will be understood. Such studies will also tell us more about what enhanced NH means for GCs in general.

D. B. and Y. L. would like to thank the telescope operators at the MMT for their help and the anonymous referee for helpful comments. D. B. thanks Eva Grebel for sending him her data on distances of Local Group galaxies.
Bajaja, E., \& Shane, W. W. 1982, A\&AS, 49, 745

Barmby, P., Huchra, J. P., Brodie, J. P., Forbes, D. A., Schroder, L. L., \& Grillmair, C. J. 2000, AJ, 119, 727

Battistini, P., Bonoli, F., Braccesi, A., Fusi-Pecci, F., Malagnini, M. L., \& Marano, B. 1980, A\&AS, 42, 357

Beasley, M., Brodie, J. P., Strader, J., Forbes, D. A., Proctor, R. N., Barmby, P., \& Huchra, J. P. 2004, AJ, submitted (astro-ph/0405009)

Behr, B. B. 2003, ApJS, 149, 67

Bergbusch, P. A., \& VandenBerg, D. A. 2001, ApJ, 556, 322

Briley, M. M., Cohen, J. G., \& Stetson, P. B. 2004, AJ, 127, 1579

Brinks, E., \& Burton, W. B. 1984, A\&A, 141, 195

Brown, T. M., Ferguson, H. C., Smith, E., Kimble, R. A., Sweigart, A. V., Renzini, A., Rich, R. M., \& VandenBerg, D. A. 2003, ApJ, 592, L17

Burstein, D., Bender, R., Faber, S. M., \& Nolthenius, R. 1997, AJ, 114, 1365

\section{REFERENCES}

Burstein, D., Faber, S. M., Gaskell, C. M., \& Krumm, N. 1984, ApJ, 287, 586 Carretta, E., Gratton, R. G., Bragaglia, A., Bonifacio, P., \& Pasquini, L. 2004, A\&A, 416, 925

Castilho, B., Pasquini, L., Allen, D., Barbuy, B., \& Molaro, P. 2000, A\&A, 361, 92

Davidge, T. J., \& Clark, C. C. 1994, AJ, 107, 946

de Vaucouleurs, G., de Vaucouleurs, A., Corwin, H. G., Jr., Buta, R. J., Paturel, G., \& Fouqué, P. 1991, Third Reference Catalogue of Bright Galaxies (New York: Springer)

Forbes, D., Strader, J., \& Brodie, J. 2004, AJ, 127, 3394

Freedman, W. L., et al. 2001, ApJ, 553, 47

Harris, W. E. 1996, AJ, 112, 1487

Harris, W. E., \& Pudritz, R. E. 1994, ApJ, 429, 177

Ibata, R. A., Gilmore, G., \& Irwin, M. J. 1994, Nature, 370, 194 
Kauffmann, G., Nusser, A., \& Steinmetz, M. 1997, MNRAS, 286, 795

Kraft, R. P., \& Ivans, I. I. 2003, PASP, 115, 143

Leonardi, A. J., \& Rose, J. A. 2003, AJ, 126, 1811

Li, Y., \& Burstein, D. 2003, ApJ, 598, L103

Ma, J., et al. 2001, AJ, 122, 1796

Morrison, H. L., Harding, P., Perrett, K., \& Hurley-Keller, D. 2004, ApJ, 603, 87

Nilson, P. 1973, Uppsala General Catalogue of Galaxies (Uppsala: Astron. Obs.)

Ponder, J. M., et al. 1998, AJ, 116, 2297
Sargent, W. L. W., Kowal, C. T., Hartwick, D. A., \& van den Bergh, S. 1977, AJ, 82, 947

Tomkin, J., \& Lambert, D. L. 1984, ApJ, 279, 220

Trager, S. C., Worthey, G., Faber, S. M., Burstein, D., \& Gonzalez, J. J. 1998, ApJS, 116, 1

Vetesnik 1962a, Bull. Astron. Inst. Czechoslovakia, 13, 180 1962b, Bull. Astron. Inst. Czechoslovakia, 13, 218

Whitmore, B. C., \& Schweizer, F. 1995, AJ, 109, 960 